\title{
The state of molecular gas in the Small Magellanic Cloud
}

\author{
Adam K. Leroy ${ }^{1}$, Alberto D. Bolatto ${ }^{2}$, Erik Rosolowsky ${ }^{3}$, \\ Snežana Stanimirović ${ }^{4}$, Norikazu Mizuno ${ }^{5}$, Caroline Bot $^{6}$, \\ Frank Israel ${ }^{7}$, Fabian Walter ${ }^{1}$ and Leo Blitz ${ }^{8}$ \\ ${ }^{1}$ Max Planck Institute for Astronomy, Heidelberg, Germany \\ ${ }^{2}$ Department of Astronomy and Laboratory for Millimeter-wave Astronomy, \\ University of Maryland, USA \\ ${ }^{3}$ Department of Mathematics, Statistics, and Physics, \\ University British Columbia at Okanagan, Canada \\ ${ }^{4}$ Department of Astronomy, University of Wisconsin, USA \\ ${ }^{5}$ Department of Astrophysics, Nagoya University, Japan \\ ${ }^{6}$ Observatoire Astronomique de Strasbourg, France \\ ${ }^{7}$ Sterrewacht Leiden, The Netherlands \\ ${ }^{8}$ Department of Astronomy and Radio Astronomy Laboratory, U.C. Berkeley, USA
}

\begin{abstract}
We compare the resolved properties of giant molecular clouds (GMCs) in the Small Magellanic Cloud (SMC) and other low mass galaxies to those in more massive spirals. When measured using CO line emission, differences among the various populations of GMCs are fairly small. We contrast this result with the view afforded by dust emission in the Small Magellanic Cloud. Comparing temperature-corrected dust opacity to the distribution of $\mathrm{H}$ I suggests extended envelopes of $\mathrm{CO}$-free $\mathrm{H}_{2}$, implying that $\mathrm{CO}$ traces only the highest density $\mathrm{H}_{2}$ in the SMC. Including this $\mathrm{CO}$-free $\mathrm{H}_{2}$, the gas depletion time, $\mathrm{H}_{2}$-to- $\mathrm{H}$ I ratio, and $\mathrm{H}_{2}$-to-stellar mass/light ratio in the SMC are all typical of those found in more massive irregular galaxies.
\end{abstract}

Keywords. ISM: clouds, galaxies: dwarf, galaxies: individual (SMC), galaxies: ISM, Magellanic Clouds

\section{Introduction}

In the Milky Way, most star formation occurs in giant molecular clouds (GMCs). Because $\mathrm{H}_{2}$ does not readily emit under the conditions in these clouds, they are usually observed via line emission from tracer molecules — most commonly the lowest rotational transition of CO. From these observations we know that most of the $\mathrm{H}_{2}$ in the Milky Way lies in gravitationally bound GMCs with masses from $10^{5}$ to $10^{6} \mathrm{M}_{\odot}$. Their luminosities, line widths, and sizes obey certain scaling relations - commonly referred to as "Larson's Laws" (Larson 1981; see reviews by, e.g., Blitz 1993 and McKee \& Ostriker 2007).

An open question is how the properties of GMCs are affected by their environment. Star formation over the history of the universe has occurred under a vast range of conditions from chemically pristine gas to violent galaxy mergers. Even "normal" disk galaxies host a wide range of radiation fields, metallicities, pressures, and dynamical states. Environment may affect the fraction of gas converted to stars (and thus stellar clustering and feedback) or the initial distribution of stellar masses. In order to do so, these conditions must first affect GMCs, the structures out of which stars form.

In particular, the relationship between metallicity (and the closely related dust abundance) and GMC structure is both intriguing and difficult to approach. There are 
fundamental reasons to expect a relationship: at all but the lowest metallicities, $\mathrm{H} \mathrm{I}$ is converted to $\mathrm{H}_{2}$ on the surface of dust grains and dust can help shield $\mathrm{H}_{2}$ from dissociating radiation. Further, metallicity impacts thermal structure of the atomic ISM, so one may expect that clouds form under different conditions in low metallicity environments. Star formation obviously does proceed at low metallicities: even in the nearby universe, there are numerous vigorously star-forming low-metallicity galaxies. Unfortunately, it is difficult to characterize molecular gas in these galaxies because their $\mathrm{CO}$ emission is faint (e.g., Taylor et al. 1998). This is partially because low metallicity galaxies also tend to have low masses (e.g., Lee et al. 2006), making them less luminous at all wavelengths. However, very low-metallicity star-forming galaxies show distinctly low normalized CO emission - i.e., their CO emission is low compared to their star formation rate (SFR) or stellar mass. Diminished dust shielding is probably as responsible for this as the underabundance of $\mathrm{C}$ and $\mathrm{O}$ (e.g., Maloney \& Black 1988). Regardless of the cause, the practical results are that it is difficult to observe molecular gas in very low metallicity systems and that the standard tracers of $\mathrm{H}_{2}$ must be employed with caution.

As the nearest low-metallicity, actively star-forming system, the Small Magellanic Cloud (SMC) is key to understand the effect of metallicity on GMC structure. Some effects are clearly present: the SMC's normalized CO emission $\left(L_{\mathrm{CO}} \sim 10^{5} \mathrm{~K} \mathrm{~km} \mathrm{~s}^{-1} \mathrm{pc}^{-2}\right.$, Mizuno et al. 2001) is quite low compared to its other properties. For example, Wilke et al. (2004) estimate the SFR in the SMC to be $\sim 0.05 \mathrm{M}_{\odot} \mathrm{yr}^{-1}$. For a standard Galactic CO-to- $\mathrm{H}_{2}$ conversion factor, this implies a molecular gas depletion time $\left(\mathrm{M}_{\mathrm{H} 2} / \mathrm{SFR}\right)$ of $\sim 10^{7}$ years. This is about two orders of magnitude lower than that observed in most spiral galaxies (e.g., Young et al. 1996, Kennicutt 1998). The implied ratio of $\mathrm{H}_{2}$-to-H I is also strikingly small, $\sim 1$-to-1000 (Stanimirović et al. 2004), about two orders of magnitude lower than that in more massive irregular galaxies (e.g., Young \& Scoville 1991). In Fig. 1, we show that the ratio of CO emission to stellar light, which varies only weakly among massive star forming galaxies is similarly low in the SMC (blue circle).

These ratios place the SMC in the company of only a few very nearby low metallicity galaxies that have observed - but very faint - CO emission. More distant analogs to these systems tend to be CO non-detections. The SMC is unique among these objects because of its proximity, which allows even single-dish millimeter-wave telescopes to achieve good spatial resolution. This has allowed extensive studies of of molecular gas on the scale of individual GMCs (e.g., Rubio et al. 1993a,b; Mizuno et al. 2001; Bolatto et al. 2003; Rubio et al. 2004; Bot et al. 2007). In these proceedings, we summarize two recent results : 1) that the properties of resolved GMCs — as measured from CO emission in the SMC and other dwarf galaxies are quite similar to those in the Milky Way, M31, and M 33; and 2) that dust emission suggests large, extended reservoirs of CO-free $\mathrm{H}_{2}$ surrounding these Galactic-looking CO clouds.

\section{GMC scaling relations in low mass galaxies}

A basic test of the state of molecular gas is to resolve $\mathrm{CO}$ emission into individual GMCs and compare their properties to those of Milky Way GMCs. Bolatto et al. (2008, B08) recently attempted this test by measuring GMC properties in 11 nearby dwarf galaxies (including the SMC) and comparing these to results from the Milky Way, M 31, and M 33. The data are a mixture of new and previously published observations $\dagger$ obtained

$\dagger$ Spirals: Milky Way, Solomon et al. (1987); M 33, Rosolowsky et al. (2003); M 31, Rosolowsky et al. (2007). Magellanic Clouds: LIRS 36 \& LIRS 49, Rubio et al. (1993a,b); N 159, Bolatto et al. (2000); N83, Bolatto et al. (2003). Local Group Dwarfs: NGC 185 and NGC 205, Young 


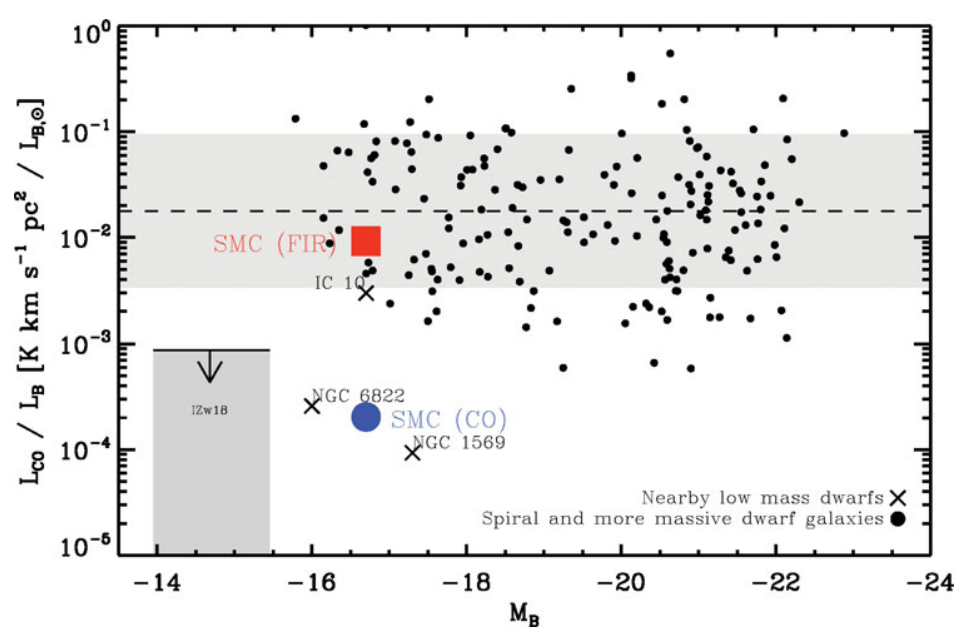

Figure 1. The ratio of $\mathrm{CO}$ to B-band luminosity vs. B-band absolute magnitude in nearby galaxies with detected CO emission (adapted from Leroy et al. 2007b). Several nearby, low-metallicity dwarfs are highlighted, including the SMC (blue circle). These systems show faint $\mathrm{CO}$ emission relative to their other properties (they also show very low $\mathrm{CO} / \mathrm{SFR}$ and $\mathrm{CO} / \mathrm{HI}$ ). We also plot the normalized $\mathrm{CO}$ content one would expect if all of the $\mathrm{H}_{2}$ inferred from dust emission exhibited a Galactic CO-to- $\mathrm{H}_{2}$ conversion factor (red square). The ratio is close to that in larger galaxies, suggesting that $\mathrm{CO}$ is more strongly affected by environmental differences than $\mathrm{H}_{2}$.

with interferometers (BIMA, OVRO, and PdBI) and single dish telescopes (SEST). The targets span metallicities from $12+\log \mathrm{O} / \mathrm{H}=8.02$ (the $\mathrm{SMC}$ ) to 8.85 (i.e., slightly above solar) and distances out to 4 Mpc. B08 measured GMC properties using the CPROPS algorithm (Rosolowsky \& Leroy 2006). CPROPS makes a conservative decomposition of emission into individual GMCs and then uses moment methods, extrapolation to ideal sensitivity, and a simple quadratic deconvolution to derive sizes, line widths, and luminosities (corrected for resolution and sensitivity). The goal of this approach is a consistent intercomparison of observations at mixed resolution and signal-to-noise. Blitz et al. (2007) recently present a complementary review and analysis (also using CPROPS) that focused on complete surveys of Local Group galaxies.

B08 compared the properties of extragalactic GMCs to the scaling relations measured for Milky Way GMCs, essentially asking whether GMCs in dwarf galaxies are consistent with being drawn from the population of Milky Way GMCs. Broadly, the answer is "yes". GMCs from the Magellanic Clouds, Local Group dwarfs, and more distant dwarfs tend to lie within a factor of $\sim 2$ of the Milky Way GMC scaling relations. That is, $C O$ emission from dwarf galaxy GMCs closely resembles that from GMCs in the Milky Way and other Local Group spirals. We show this in Fig. 2, which presents GMC line width as a function of size (left) and virial mass as a function of CO luminosity (right). Similarities in the line width-size relation may reflect a similar character of turbulence in all of the systems surveyed. The correlation between virial mass and CO luminosity is often interpreted to mean that GMCs are in approximate virial equilibrium and with CO luminosity a good tracer of cloud mass. A particularly surprising result is that low metallicity GMCs (e.g., from the SMC or IC 10) show roughly the same virial mass-to-luminosity relation seen in

et al. (2001); IC 10, Walter et al. (2003), Leroy et al. (2006). Dwarfs Beyond the Local Group: NGC 1569, Taylor et al. (1999); NGC 4214, Walter et al. (2001), Bolatto et al. (2008); NGC 4605, Bolatto et al. (2002); NGC 3077, Walter et al. (2002), Bolatto et al. (2008); NGC 4449, Bolatto et al. (2008); NGC 2976, Simon et al. (2003). 

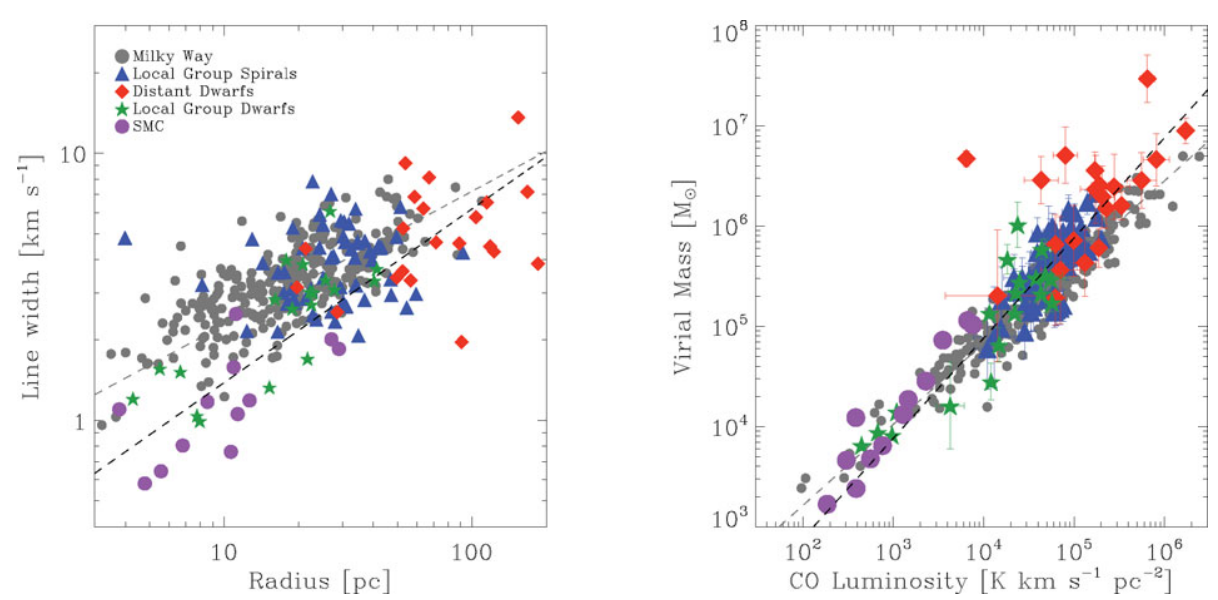

Figure 2. GMC scaling relations in the Milky Way and other galaxies (adapted from Bolatto et al. 2008): (left) line width vs. cloud radius and (right) virial mass $\left(\propto R \sigma^{2}\right)$ vs. CO luminosity. Milky Way clouds are light gray circles, M 31 and M 33 are blue triangles, Local Group dwarfs are green stars, and more distant dwarfs are red diamonds. SMC clouds are purple circles.

more massive galaxies. If GMCs are virialized, this implies that the CO-to- $\mathrm{H}_{2}$ conversion factor for resolved, CO-bright clumps has little dependence on metallicity.

Despite overall agreement, B08 do find some differences among the populations surveyed. For example, the left panel in Fig. 2 shows that SMC clouds have lower line widths than Galactic clouds of the same size. Possible explanations are increased magnetic support in SMC clouds (e.g., Bot et al. 2007) or a simple lack of virial equilibrium (implying short-lived GMCs). An alternative explanation is that the Milky Way scaling relations derived by Solomon et al. (1987) are in need of revision: Heyer et al. (2008) recently re-measured the properties of the Solomon et al. clouds using the Galactic Ring Survey (Jackson et al. 2006) and found a relationship among size, line width, and surface density that agrees well with the B08 data.

\section{3. $\mathrm{H}_{2}$ traced by dust in the SMC}

Thus GMC properties measured from CO suggest that molecular gas in the SMC is similar to that in the Galaxy (with perhaps small differences). However, as we have already emphasized, $\mathrm{CO}$ is a suspect tracer of molecular gas at low metallicities. Israel (1997) used IRAS emission (dust continuum) to trace molecular gas in dwarf irregulars and found evidence for large amounts of $\mathrm{CO}$-free $\mathrm{H}_{2}$. His study was limited to relatively large scales by the available data. Subsequently, improved surveys of the SMC have been carried out in CO (Mizuno et al. 2001), H I (Stanimirović et al. 2004), and the infrared (the Spitzer Survey of the SMC, Bolatto et al. 2007). In Leroy et al. (2007a), we combined these data to use dust as a probe of the $\mathrm{H}_{2}$ distribution.

Our technique was to estimate the surface density of dust, $\Sigma_{\text {Dust }}$, everywhere in the SMC using 100 and $160 \mu \mathrm{m}$ emission (two or more bands are needed to account for varying dust temperature). We then identified likely $\mathrm{H}_{2}$ peaks from $\mathrm{CO}$ and measured the dustto-gas ratio (DGR) near these peaks, but displaced enough that $\mathrm{H} \mathrm{I}$ (and not $\mathrm{H}_{2}$ ) is likely to dominate the ISM. We combined these locally measured DGRs with the $\Sigma_{\text {Dust }}$ near molecular peaks to estimate the total gas surface density. By subtracting the measured contribution of $\mathrm{H}$ I, we derived a dust-based map of $\mathrm{H}_{2}$, i.e., $\Sigma_{\mathrm{H} 2}=D G R^{-1} \times \Sigma_{\mathrm{Dust}}-\Sigma_{\mathrm{HI}}$.

This approach yields $\sim 3 \times 10^{7} \mathrm{M}_{\odot}$ of $\mathrm{H}_{2}$, much higher than using $\mathrm{CO}$ alone. This is close to what one would infer from the SMC's other properties: the implied $\mathrm{H}_{2}$ depletion 

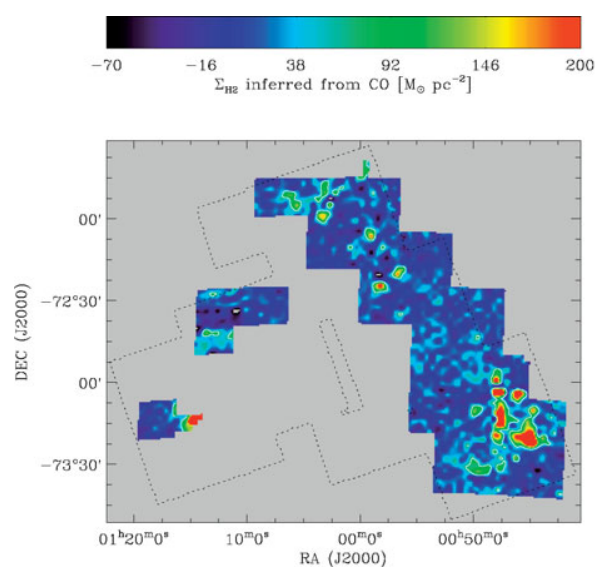
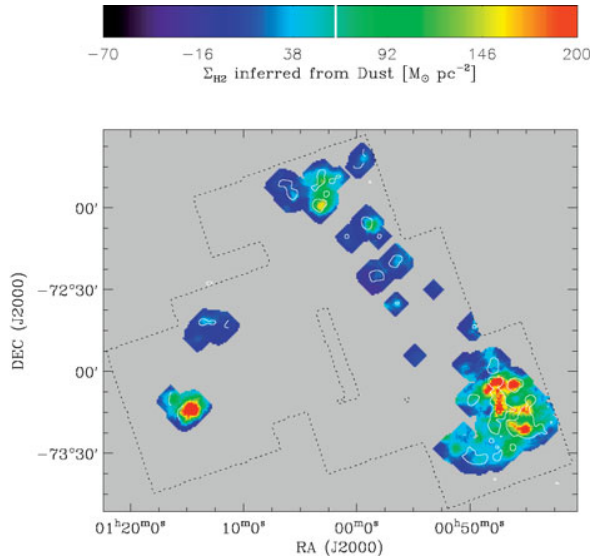

Figure 3. Mass surface density of $\mathrm{H}_{2}$ as inferred from $\mathrm{CO}$ (Mizuno et al. 2001, left) and dust emission (right). $\mathrm{CO}$ has been translated to $\mathrm{H}_{2}$ surface density using the average CO-to- $\mathrm{H}_{2}$ conversion factor implied by the dust map (so that the two maps contain the same total mass). $\mathrm{H}_{2}$ inferred from dust shows the same peaks as $\mathrm{CO}$, but has a more extended distribution. The overall mass of $\mathrm{H}_{2}$ implied by dust, $\sim 3 \times 10^{7} \mathrm{M}_{\odot}$, is also much larger than one would infer from $\mathrm{CO}$ emission alone $\left(\sim 5 \times 10^{5} \mathrm{M}_{\odot}\right.$ for a Galactic CO-to- $\mathrm{H}_{2}$ conversion factor; see Fig. 1$)$.

time is $\sim 6 \times 10^{8}$ years, within a factor of $\sim 2-3$ of that found for spirals (i.e., the SMC obeys approximately the same "molecular Schmidt law" as larger galaxies). The implied $\mathrm{H}_{2}$-to-H I ratio is $\sim 1: 10$, consistent with larger irregulars, and the ratio of $\mathrm{H}_{2}$ to B-band luminosity also matches that in larger galaxies (red square in Fig. 1).

This result is quite distinct from what B08 find using $\mathrm{CO}$ and the detailed distribution of $\mathrm{H}_{2}$ compared to $\mathrm{CO}$ suggest a possible reason. Although $\mathrm{H}_{2}$ and $\mathrm{CO}$ share roughly the same peaks, we find a distribution of $\mathrm{H}_{2}$ that is more extended than that of $\mathrm{CO}$ (compare the left and right panels in Fig. 3). Indeed, about several $\mathrm{H}_{2}$ peaks, we measure $\mathrm{H}_{2}$ to be more extended than $\mathrm{CO}$ by a factor of $\sim 1.5$ in radius (a number revised from Leroy et al. 2007 to include the effects of H I opacity estimated by Dickey et al. 2000; also likely a lower limit given the relatively large physical size of the NANTEN beam). This difference suggests the selective photodissociation of CO in SMC clouds (Maloney \& Black 1988), i.e. that in the outer parts of clouds $\mathrm{H}_{2}$ self-shields while $\mathrm{CO}$ - which relies largely on dust for shielding - is destroyed by dissociating radiation.

If $\mathrm{CO}$ is preferentially destroyed in the outer parts of SMC clouds, then our conclusions may not be contradictory at all. The similarity in GMC properties measured from CO implies that the densest parts of these clouds resemble entire Milky Way GMCs. Similar situations are already observed in Milky Way clouds: the line width-size relation appears to extend from the scale of whole clouds down to less than a parsec (Heyer \& Brunt 2004; Rosolowsky et al. 2008) and substructures within Milky Way GMCs can appear virialized (e.g., Rosolowsky et al. 2008). In this case the intercloud dispersion (rather than the CO line width of individual clouds) may offer an independent way to trace the full molecular mass and indeed measurements of SMC clouds by Rubio et al. (1993b) and Bolatto et al. (2003) find the ratio of CO luminosity to virial mass to be a strong function of scale. That CO-free $\mathrm{H}_{2}$ is needed to establish rough agreement between $\mathrm{H}_{2}$ depletion times in the SMC and larger galaxies is also not as surprising as it may first appear. In the Milky Way, little or no star formation is actually associated with the bulk 
of CO emission; instead, it tends to occur only towards the highest density/extinction peaks (e.g., Johnstone et al. 2004).

\section{References}

Blitz, L. 1993, in Protostars and Planets III, p. 125

Blitz, L., Fukui, Y., Kawamura, A., Leroy, A., Mizuno, N., \& Rosolowsky, E. 2007, in B. Reipurth, D. Jewitt, \& K. Keil (eds.), Protostars and Planets $V$ (Tucson: University of Arizona Press), p. 81

Bolatto, A. D., Jackson, J. M., Israel, F. P., Zhang, X., \& Kim, S. 2000, ApJ, 545, 234

Bolatto, A. D., Simon, J. D., Leroy, A., \& Blitz, L. 2002, ApJ, 565, 238

Bolatto, A. D., Leroy, A., Israel, F. P., \& Jackson, J. M. 2003, ApJ, 595, 167

Bolatto, A. D., Simon, J. D., Stanimirović, S., et al. 2007, ApJ, 655, 12

Bolatto, A. D., Leroy, A. K., Rosolowsky, E., Walter, F., \& Blitz, L. 2008, ApJ, 686, 948

Bot, C., Boulanger, F., Rubio, M., \& Rantakyro, F. 2007, AESA, 471, 103

Dickey, J. M., Mebold U., Stanimirović, S., \& Staveley-Smith, L. 2000, ApJ, 536, 756

Heyer, M. H. \& Brunt, C. M. 2004, ApJ, 615, L45

Heyer, M., Krawczyk, C., Duval, J., \& Jackson, J. M. 2008, ApJ submitted

Israel, F. P. 1997, A\& A, 328, 471

Jackson, J. M., Rathborne, J. M., Shah, R. Y., et al. 2006, ApJS, 163, 145

Johnstone, D., Di Francesco, J., \& Kirk, H. 2004, ApJ, 611, L45

Kennicutt, R. C., Jr. 1998, ApJ, 498, 541

Larson, R. B. 1981, MNRAS, 194, 809

Lee, H., Skillman, E. D., Cannon, J. M., Jackson, D. C., Gehrz, R. D., Polomski, E. F., \& Woodward, C. E. 2006, ApJ, 647, 970

Leroy, A., Bolatto, A., Walter, F., \& Blitz, L. 2006, ApJ, 643, 825

Leroy, A., Bolatto, A., Stanimirović, S., Mizuno, N., Israel, F., \& Bot, C. 2007a, ApJ, 658, 1027

Leroy, A., Cannon, J., Walter, F., Bolatto, A., \& Weiss, A. 2007b, ApJ, 663, 990

Maloney, P. \& Black, J. H. 1988, ApJ, 325, 389

McKee, C. F. \& Ostriker, E. C. 2007, ARAA, 45, 565

Mizuno, N., Rubio, M., Mizuno, A., Yamaguchi, R., Onishi, T., \& Fukui, Y. 2001, PASJ, 53, L45

Simon, J. D., Bolatto, A. D., Leroy, A., \& Blitz, L. 2003, ApJ, 596, 957

Solomon, P. M., Rivolo, A. R., Barrett, J., \& Yahil, A. 1987, ApJ, 319, 730

Stanimirović, S., Staveley-Smith, L., \& Jones, P. A. 2004, ApJ, 604, 176

Rosolowsky, E., Engargiola, G., Plambeck, R., \& Blitz, L. 2003, ApJ, 599, 258

Rosolowsky, E. \& Leroy, A. 2006, PASP, 118, 590

Rosolowsky, E. 2007, ApJ, 654, 240

Rosolowsky, E. W., Pineda, J. E., Kauffmann, J., \& Goodman, A. A. 2008, ApJ, 679, 1338

Rubio, M., Lequeux, J., Boulanger, F. et al. 1993a, A\&A, 271, 1

Rubio, M., Lequeux, J., \& Boulanger, F. 1993b, A\&SA, 271, 9

Rubio, M., Boulanger, F., Rantakyro, F., \& Contursi, A. 2004, A\&A, 425, L1

Taylor, C. L., Kobulnicky, H. A., \& Skillman, E. D. 1998, AJ, 116, 2746

Taylor, C. L., Hüttemeister, S., Klein, U., \& Greve, A. 1999, A\&A, 349, 424

Walter, F., Taylor, C. L., Hüttemeister, S., Scoville, N., \& McIntyre, V. 2001, AJ, 121, 727

Walter, F., Weiss, A., Martin, C., \& Scoville, N. 2002, AJ, 123, 225

Walter, F. 2003, in Star Formation at High Angular Resolution, IAU Symposium 221, p. 176

Wilke, K., Klaas, U., Lemke, D., Mattila, K., Stickel, M., \& Haas, M. 2004, A\&\&A, 414, 69

Young, J. S. \& Scoville, N. Z. 1991, ARAA, 29, 581

Young, J. S., Allen, L., Kenney, J. D. P., Lesser, A., \& Rownd, B. 1996, AJ, 112, 1903

Young, L. M. 2001, AJ, 122, 1747 